\title{
Curcumin inhibits EMMPRIN and MMP-9 expression through AMPK-MAPK and PKC signaling in PMA induced macrophages
}

\author{
Jiatian Cao ${ }^{1 \dagger}$, Zhihua Han ${ }^{1 \dagger}$, Lei Tian $^{1 \dagger}$, Kan Chen ${ }^{1}$, Yuqi Fan', Bozhi Ye ${ }^{2}$, Weijian Huang ${ }^{2}$, Changqian Wang ${ }^{1 *}$ \\ and Zhouqing Huang ${ }^{2^{*}}$
}

\begin{abstract}
In coronary arteries, plaque disruption, the major acute clinical manifestations of atherosclerosis, leads to a subsequent cardiac event, such as acute myocardial infarction (AMI) and unstable angina pectoris (UA). Numerous reports have shown that high expression of MMP-9 (matrix metalloproteinase-9), MMP-13 (matrix metalloproteinase-13) and EMMPRIN (extracellular matrix metalloproteinase induce) in monocyte/macrophage results in the plaque progression and destabilization. Curcumin exerts well-known anti-inflammatory and antioxidant effects and probably has a protective role in the atherosclerosis. The purpose of our study was to investigate the molecular mechanisms by which curcumin affects MMP-9, MMP13 and EMMPRIN in PMA (phorbol 12-myristate 13-acetate) induced macrophages. Human monocytic cells (THP-1 cells) were pretreated with curcumin or compound $\mathrm{C}$ for $1 \mathrm{~h}$, and then induced by PMA for $48 \mathrm{~h}$. Total RNA and proteins were collected for real-time PCR and Western blot analysis, respectively. In the present study, the exposure to curcumin resulted in attenuated JNK, p38, and ERK activation and decreased expression of MMP-9, MMP-13 and EMMPRIN in PMA induced macrophages. Moreover, we demonstrated that AMPK (AMP-activated protein kinase) and PKC (Protein Kinase C) was activated by PMA during monocyte/macrophage differentiation. Furthermore, curcumin reversed PMA stimulated PKC activation and suppressed the chronic activation of AMPK, which in turn reduced the expression of MMP-9, MMP-13 and EMMPRIN. Therefore, it is suggested that curcumin by inhibiting AMPK-MAPK (mitogen activated protein kinase) and PKC pathway may led to down-regulated EMMPRIN, MMP-9 and MMP-13 expression in PMA-induced THP-1 cells.
\end{abstract}

Keywords: Curcumin, EMMPRIN, MMP-9, MMP-13, AMPK, MAPK, Atheroslerosis

\section{Background}

EMMPRIN, also termed CD147 or M6 antigen, is a 58$\mathrm{kDa}$ cell surface glycoprotein described first in tumor cells. It participates in numerous physiological processes, play a central role in tumor metastasis, cell adhesion, angiogenesis, chemoresistance and atherosclerosis [1,2]. EMMPRIN has been reported to stimulates secretion of MMP-9 (matrix metalloproteinase-9) in monocytes [1,3], have strong positive correlation with MMP13 [4,5] or several MMPs in other cells [6,7], and activates MMP-9

\footnotetext{
* Correspondence: changqianwang@hotmail.com; susiehzq@126.com ${ }^{\dagger}$ Equal contributors

'Division of Cardiology, Shanghai Ninth People's Hospital Affiliated Shanghai Jiaotong University School of Medicine, Shanghai, P. R. China

${ }^{2}$ Division of Cardiology, the First Affiliated Hospital of Wenzhou Medical University, Wenzhou, Zhejiang, P. R. China
}

in atherosclerotic plaque [8]. MMP-9 belongs to a family of zinc- and calcium-dependent endopeptidases. It is a $92 \mathrm{kDa}$ protein that regulates numerous cell activities, involving in various physiological functions, such as cellcell contact, tissue remodeling cell migration and cellular differentiation [9]. Recent data showed that increased EMMPRIN expression affects plaque stability $[1,8]$, and accelerates the transition from a stable plaque to an unstable plaque in atherogenic cells, such as monocytes/ macrophages and coronary smooth muscle cells $[10,11]$. Despite recent advance in drug treatment and surgical therapies, atherosclerosis remains to be a major cause of death throughout the world. In coronary arteries, plaque disruption is the majority of acute clinical manifestations of atherosclerosis, leading to a subsequent cardiac event, such as AMI and UA. Monocyte-derived macrophages 
are known to play a critical role in the initiation and progression of atherosclerosis. Over-expression of MMP-9 and EMMPRIN in monocytes/macrophages results in plaque progression and destabilization $[6,12]$. Plaque rupture is thought to result from the degradation of extracellular matrix components by macrophage-derived matrix metalloproteinases (MMPs) [13]. Numerous reports have shown that MMP-9 is one of the most important MMPs contributing to plaque rupture, and its expression level is induced in serious coronary atherosclerosis and AMI and UA [14]. In addition, MMP-9 induces acute plaque disruption in Apoe-/-mice $[15,16]$. Previous reports demonstrated that MMP-13 is involved in atherogenesis and decreasing plaque stability [17]. MMP-13 might be overexpressed in both human and experimental atherosclerosis as well $[18,19]$. All these data indicate that EMMPRIN-mediated MMPs induction is involved in the process of atherosclerotic lesion. Base on these pieces of evidence, we hypothesized that agents suppressing EMMPRIN and MMP-9 expression would be potential therapeutic agents that ameliorate the development of atherosclerosis. All these data indicate that EMMPRIN-mediated MMP induction is involved in the process of atherosclerotic lesion. Based on these pieces of evidence, we hypothesized that agents suppressing EMMPRIN and MMP-9 expression would be potential therapeutic agents that ameliorate the development of atherosclerosis.

During past few years, accumulating evidence has suggested that curcumin has significant inhibitory effect on MMPs in cancer, arthritis and ulcer [20]. Curcumin (diferuloylmethane), a polyphenol derived from turmeric and curcuma longa, is a pharmacologically safe and effective agent that plays an important role in anti-cancer and anti-inflammatory processes. In atherosclerosis, curcumin suppresses oxLDL (oxidized low-density lipoprotein) induced CD36 expression via inhibiting p38 MAPK phosphorylation [21], and prevents the decrease of thrombospondin-4 expression in oxLDL treated murine macrophages [21]. Curcumin inhibits the adhesion of monocytes to endothelial cells [22], and reduces the migration of HASMCs (human aortic smooth muscle cells) by suppressing MMP-9 expression through downregulation of NF- $\mathrm{kB}$-dependent pathways [23]. Furthermore, in vivo data showed that curcumin inhibits atherosclerosis in $\mathrm{ApoE}(-/-)$ mice [22], and blocks the development of atherosclerosis in ApoE/LDLR-/-mice [24]. Although some studies have suggested the antiatherosclerosis activity of curcumin, the mechanism by which curcumin regulates MMP-9, MMP-13 and EMMPRIN is currently unknown. The purpose of this study was to uncover the mechanism by which curcumin regulates EMMPRIN, MMP-9 and MMP-13expression during monocyte differentiation.

\section{Materials and methods \\ Cell culture}

Human monocytic cell line THP-1 was obtained from American Type Culture Collection (ATCC, Rockville, MD, USA) and maintained at a density of $10^{6} / \mathrm{ml}$ in RPMI 1640 medium containing 10\% FBS, $10 \mathrm{mM}$ HEPES (Sigma) and $1 \%$ pen/strep solution at $37^{\circ} \mathrm{C}, 5 \% \mathrm{CO}_{2}$ incubator. Cells were cultured in six-well plates for $48 \mathrm{~h}$ in the presence of 100 nM PMA, which allowed them to differentiate into adherent macrophages [25]. Cells were pretreated with curcu$\min (0$ to $50 \mu \mathrm{M}$, Sigma, USA) or $10 \mu \mathrm{M}$ Compound C (AMPK inhibitor), PD98059(MAPKK inhibitor), SB203580 (p38 MAPK inhibitor), and SP600125(JNK inhibitor) MAP kinase inhibitors (Sigma, USA) for 1 hour, and then stimulated with PMA for another 48 hours.

\section{Cytotoxicity assay}

PMA-induced macrophages were seeded in 96-well plates at $6 \times 10^{3}$ cells/well. Twenty four hours later, cells were incubated with curcumin $(0$ to $100 \mu \mathrm{M})$ for $48 \mathrm{~h}$. Cells without any treatment were used as a control. CCK8 assay (WST-8, Dojindo, Kumamoto, Japan) was used to assess the cytotoxicity of curcumin on PMA-induced macrophages, based on the manufacturer's recommendation.

\section{Protein isolation and Western blot analysis}

Protein isolation and Western blot analysis of cell lysates were performed as previously described [26]. Briefly, membranes were first probed with primary antibodies for MMP-13(Proteintch,USA), EMMPRIN, PKC $\alpha$, PKC 11 (Life Technologies, USA), MMP-9, phospho-ERK, ERK, phospho-p38, p38, phospho-JNK, JNK, AMPK, pAMPK $\alpha$ (Cell Signaling Technology, Boston, MA) (1:1000 dilution in TBST), or $\beta$-actin (1:5000 dilution in TBST), then incubated with anti-Rabbit or anti-mouse secondary antibodies (Cell Signaling Technology, Boston, MA), followed by incubation with antibody labeled with far-red fluorescent Alexa Fluor 680 dye. All signals were detected by the Odyssey imaging system (Li-cor, USA) and data were normalized based on the $\beta$-actin level.

\section{RNA isolation, CDNA synthesis and real-time PCR}

Total RNA was extracted from PMA-induced macrophages using Trizol reagent (Invitrogen) according to the manufacturer's instructions. cDNA was synthesized using the Reverse Transcription Kit (Takara) before Real-time polymerase chain reactions were performed by SYBR Premix Ex Taq Kit (TaKaRa Code DRR041) according to the instructions . The PCR reactions were performed in duplicate and detected by the ABI-7500 Sequence Detection System (USA). The primer sequences are listed in Table 1. All results were normalized against the GAPDH level. 
Table 1 Primers used in realtime-PCR to measure the mRNA expression of MMP-9, MMP-13 and EMMPRIN

\begin{tabular}{|c|c|c|}
\hline \multicolumn{3}{|c|}{ Primer for realtime-PCR } \\
\hline Genes & Forward $\left(5^{\prime}-3^{\prime}\right)$ & Reverse $\left(5^{\prime}-3^{\prime}\right)$ \\
\hline MMP-9 & TGACGCCGCTCACCTTCACT & CGCGCCATCTGCGTTTCCAA \\
\hline MMP-13 & CATTTGATGGGCCCTCTGGCCTGC & GTTIAGGGTTGGGGTCTTCATCTC \\
\hline EMMPRIN & TTGGAGGTTGTAGGACCGGCGA & TGGGACCCTGCCCTTCAAACCA \\
\hline GAPDH & CCGCATCTTCTITTGCGTCGCC & TCTCAGCCTTGACGGTGCCA \\
\hline
\end{tabular}

\section{Gelatin zymography}

Cells in the logarithmic phase were seeded in 6-well plate at the density of $3 \times 10^{5}$ cells per well. After incubated in serum-free medium with or without curcumin $(6.25,25$ and $50 \mu \mathrm{M})$ for 1 hour, cells were incubated with $100 \mu \mathrm{M}$ PMA for another $48 \mathrm{~h}$. culture supernatants were collected, $10 \mu \mathrm{l}$ aliquots of the culture supernatant were loaded onto a $10 \%$ polyacrylamide gel containing $1 \mathrm{mg} / \mathrm{ml}$ gelatin. After electrophoresis, gels were washed twice with $2.5 \%$ Triton $\mathrm{X}-100\left(37^{\circ} \mathrm{C}\right.$, $15 \mathrm{~min}$ ) and then gels were incubated at $37^{\circ} \mathrm{C}$ for $11 \mathrm{~h}$ in developing buffer containing $10 \mathrm{mM}$ Tris Base, $40 \mathrm{mM}$ Tris-HCl, $200 \mathrm{mM} \mathrm{NaCl}, 10 \mathrm{mM} \mathrm{CaCl}$, 0.02\% Brij 35. Gels were subsequently stained with $0.5 \%$ (w/v) Coomassie Blue R-250 for $2 \mathrm{~h}$ followed by destaining with a solution containing 50\% methanol, $10 \%$ glacial acetic acid, 40\% water. MMP-9-digested regions were visualized as light bands against a dark background. An image of each gel was detected by an Odyssey imaging system (Li-cor, USA).

\section{Statistical analysis}

Data were presented as mean \pm S.D and analyzed by oneway ANOVA. $P<0.05$ was considered statistically significant. All experiments were performed at least three times.

\section{Results}

The cytotoxicity effect of curcumin on cells

To evaluate the cytotoxicity of curcumin on PMAinduced macrophages, cells were treated with 5, 10, 25, 50, 75 and $100 \mu \mathrm{M}$ curcumin for $48 \mathrm{~h}$, and then cell viability was detected by CCK-8 assay. As shown in Figure 1A, low-dose curcumin $(\leq 50 \mu \mathrm{M})$ did not significantly $(<10 \%)$ affect the cell viability. Therefore, cells were treated with dose less than $50 \mu \mathrm{M}$ for no more than 48 hours in subsequent experiments.

\section{Curcumin reduces MMP-9, MMP13 expression and MMP-9 activity}

Elevated MMP-9 expression level was previously reported during the monocyte differentiation to macrophages, while MMP-13 expression level was unknown. To determine whether curcumin has any effect on MMP-9 and MMP13 during the cell differentiation, THP-1 cells were pretreated with the indicated concentration of curcumin for
$1 \mathrm{~h}$, followed by incubating with $100 \mathrm{nM}$ PMA for $48 \mathrm{~h}$. Our results showed that curcumin significantly inhibited the upregulation of MMP-9 and MMP-13 induced by PMA, at both protein and mRNA levels, in a dosedependent manner (Figure 1B-D). Because MMP-9 is reported to remarkably enhance elastin degradation in vitro and induce plaque rupture in vivo $[15,27]$, we examined the effect of curcumin on MMP-9 enzymatic activity by SDS-polyacrylamide gelatin zymography assay. As previously reported, after overnight in-gel digestion, the gelatincontaining gel stained with coomassie blue showed an unstained transparent band at approximate $92 \mathrm{KDa}$, which was corresponding to the theoretical size of gelatin digested by MMP-9 [28]. In THP-1-derived macrophages, curcumin inhibited MMP-9 activity in a dose-dependent manner, as evidenced by gelatin zymography assay (Figure 2A,B). All the above data suggested that curcumin reduced MMP13, MMP-9 expression and MMP-9 activity in a dosedependent manner.

\section{Curcumin reduces EMMPRIN expression in a dose-dependent manner}

EMMPRIN is the major and most characterized cell surface regulator of MMP-9 and MMP-13 production $[11,12,18,19]$. Since curcumin apparently down-regulates MMP-9 and MMP-13 expression in PMA-induced macrophages, we next tested whether the inhibitory effect of curcumin on MMP-9 and MMP-13 expression was due to the inhibition of EMMPRIN expression in PMA-induced macrophages. Indeed, our results showed that EMMPRIN expression was suppressed by curcumin in a dose-dependent manner at both protein and mRNA level (Figure 1B-D), suggesting that the down-regulation of EMMPRIN by curcumin is, at least in part, responsible for the reduction of MMP-9 expression in PMA-induced macrophages.

Curcumin inhibits chronic AMPK activation induced by PMA We further tested whether AMPK activation was involved in inhibiting MMP-9 and EMMPRIN expression by curcumin. Cells were pretreated with different doses of curcumin for 1 hour and induced with PMA for another 48 hours, then the phosphorylation of AMPK $\alpha$ and total AMPK $\alpha$ was examined by Western blot. As shown in Figure 2C-D, the total AMPK increased slightly in the PMA group and curcumin can attenuates upregulation of total AMPK 


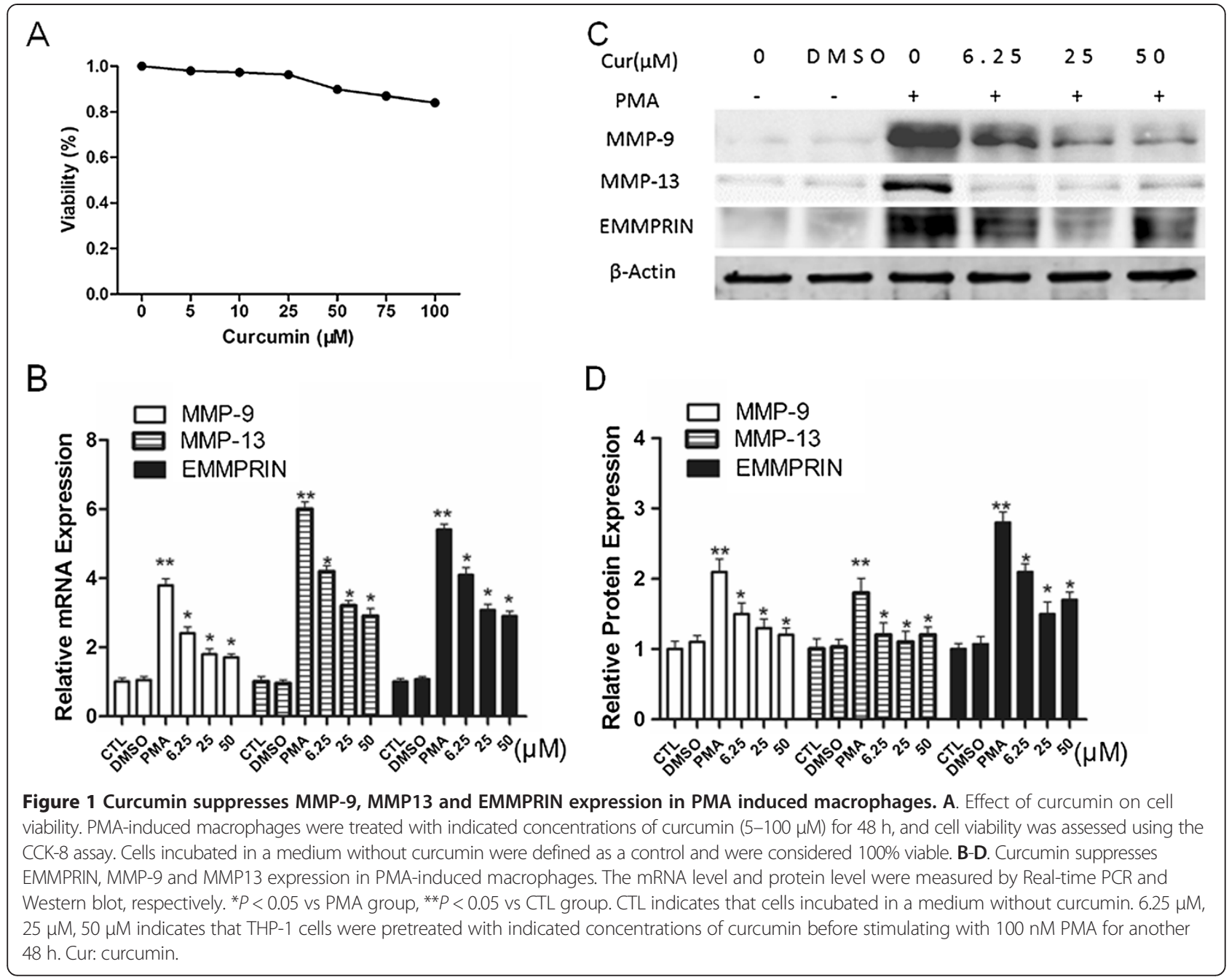

protein.PMA induced the sustained activation of AMPK $\alpha$ in THP-1 cells. Importantly, curcumin remarkably abolished AMPK $\alpha$ activation in a dose-dependent manner.

\section{Curcumin suppresses MAPK and PKC pathways in PMA -induced THP-1 cells}

Previous studies from other groups and our group indicate that PMA promotes the level of EMMPRIN and MMP-9 through activating MAPK signaling pathways $[8,29,30]$. PMA also is a strong inducer of protein kinase $\mathrm{C}$, pkc signal paly a role during PMA induced cell differentiation and adhension $[31,32]$. Thus, we wondered whether the reduced EMMPRIN expression was through the MAPK or PKC pathway. To test this hypothesis, THP-1 cells were first pretreated with curcumin for 1 hour before incubating with PMA for another 48 hours. Western data showed that curcumin significantly inhibited the phosphorylation of ERK1/ 2, p38 MAPK, JNK and PKC $\alpha, P K C \beta 1$ induced by PMA (Figure 3A-B). To further explore which MAPK signaling involved in the upregulation of MMP-9, MMP13 and
EMMPRIN in PMA induces THP-1 cell. We next examine the expression of them after treated with ERK1/2-specific inhibitor (PD98059), p38-specific inhibitor (SB203580), and JNK-specific inhibitor (SP600125).As shown in Figure 4, ERK1/2 and JNK-specific inhibitor significantly downregulated MMP-9 expression, and activation ,and p38-specific inhibitor showed weaker function. ERK1/2 and p38-specific inhibitor inhibitor significantly decreased EMMPRIN expression, whereas JNK specific inhibitor showed no inhibitory effect (Figure 4A,C). For MMP-13, ERK1/2, p38 and JNKspecific inhibitor at high dose showed remarkable inhibitory effect(Figure 4B,D). In conclusion, our result suggest that MAPK signaling and PKC pathways are involved in the regulation of EMMPRIN, MMP-9 and MMP-13 expression.

\section{Curcumin suppresses MMP-9 and EMMPRIN expression} by inhibiting phosphorylation of AMPK through MAPK pathways

To further elucidate whether AMPK has an effect on MAPK pathway after cells exposed to curcumin, we first 


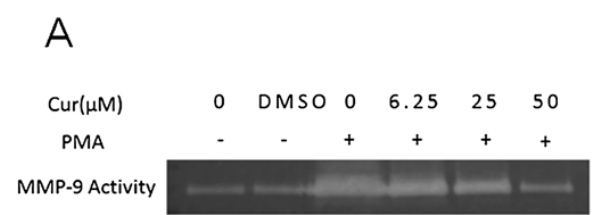

B

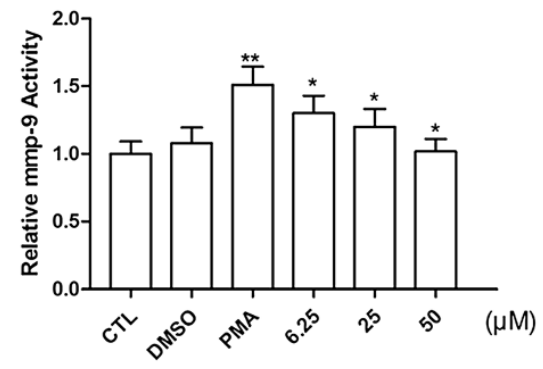

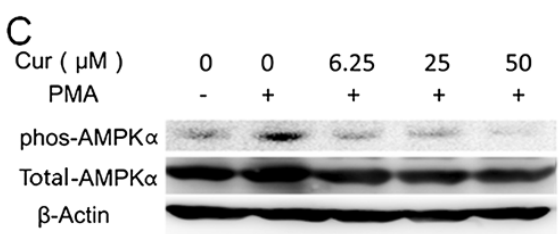

D

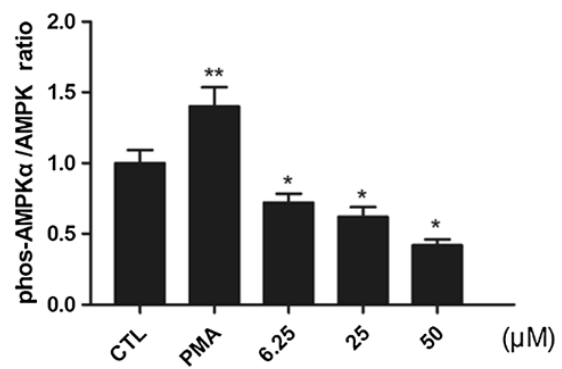

Figure 2 Curcumin suppresses the activation of MMP-9 and chronic AMPK activation. A-B. Effect of curcumin on the activity of MMP-9 from cultured supernatant. THP-1 cells were pretreated with curcumin before stimulating with PMA (100 nM) for another 48 h. MMP-9 activities were detected by gelatin zymography assay (A), and the corresponding densitometric measurement was shown in B. Cur: curcumin. C-D. Effect of curcumin on total AMPK and phospho-AMPKa (Thr172). Differentiated THP-1 cells were treated with indicated agents and assayed by Western blot using indicated antibodies $(\mathbf{C})$, and the corresponding densitometric measurement was shown in $\mathbf{D} .{ }^{*} P<0.05$ vs PMA group, ${ }^{* *} P<0.05$ vs CTL group.

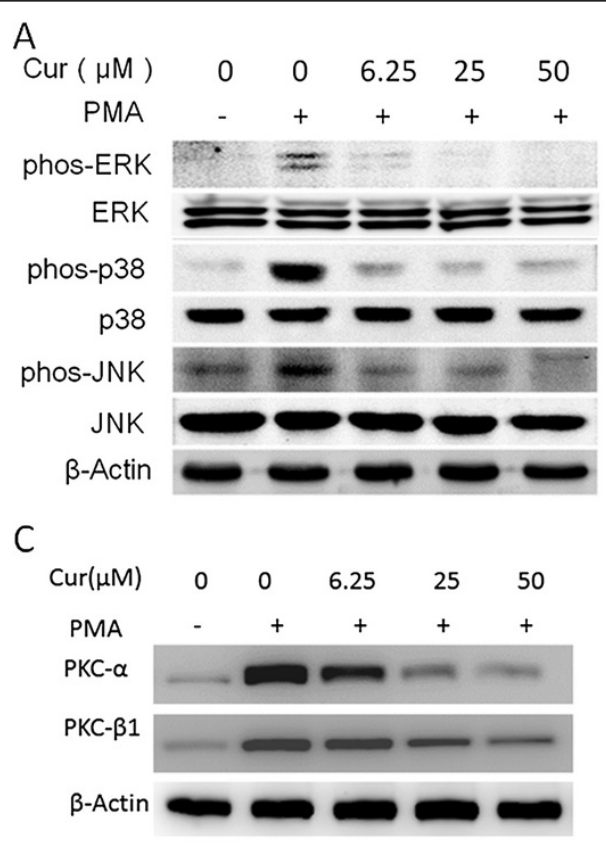

B
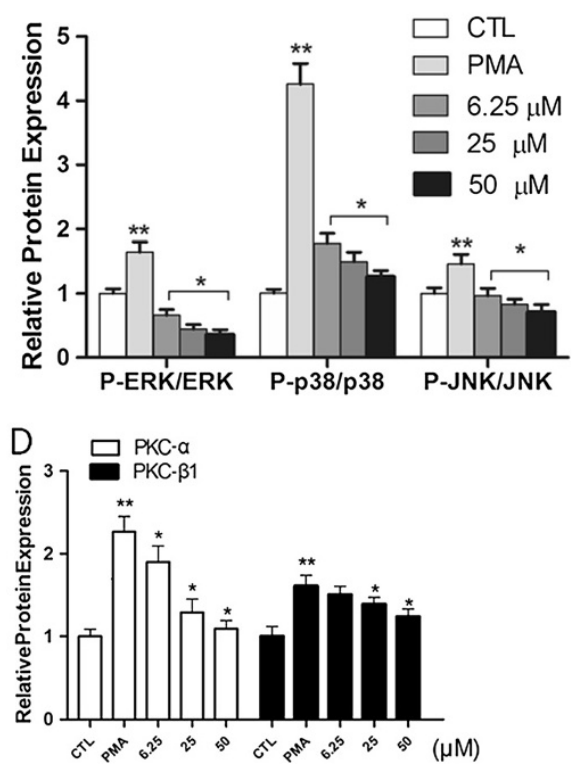

Figure 3 Curcumin inhibits JNK, ERK, and p38 phosphorylation and PKC pathway. A. Differentiated THP-1 cells were treated with indicated agents, and assayed by Western blot using indicated antibodies. Cells were pretreated with vehicle or curcumin at the indicated concentration $(5-50 \mu \mathrm{M})$ for $1 \mathrm{~h}$, followed by PMA for $48 \mathrm{~h}$. B. Protein quantification was carried out by densitometric analysis. Normalized proteins of JNK, p-JNK, ERK, p-ERK, p38 and p-p38 were normalized based on the internal control $\beta$-actin. C. Expression of PKC-a and PKC- $\beta 1$,cells were pretreated with vehicle or curcumin at the indicated concentration $(5-50 \mu \mathrm{M})$ for $1 \mathrm{~h}$, followed by PMA for $48 \mathrm{~h}$. D. Densitometry measurements of protein analysis. The mean density values of PKC-aand PKC- $\beta 1$ are expressed as ratios relative to that of $\beta$-actin. ${ }^{*} P<0.05$ vs PMA group, ${ }^{*} P<0.05$ vs $C T L$ group. 

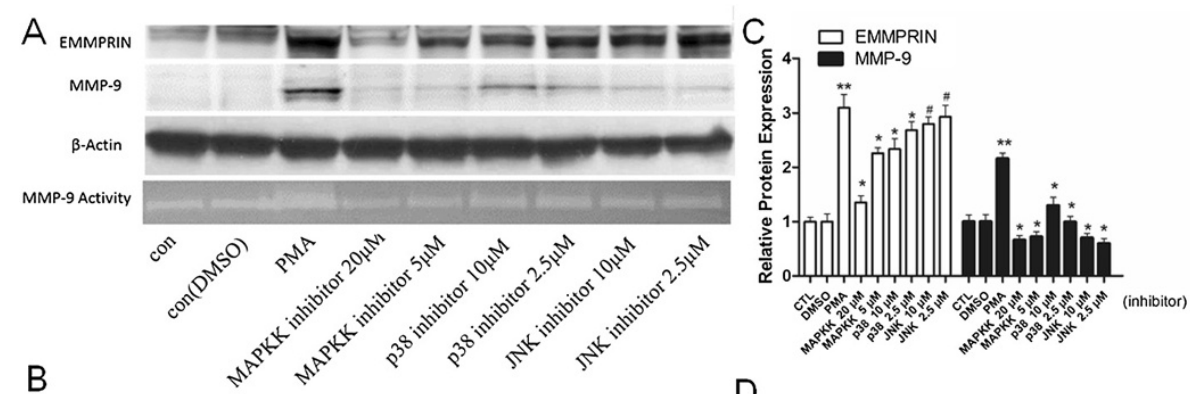

D
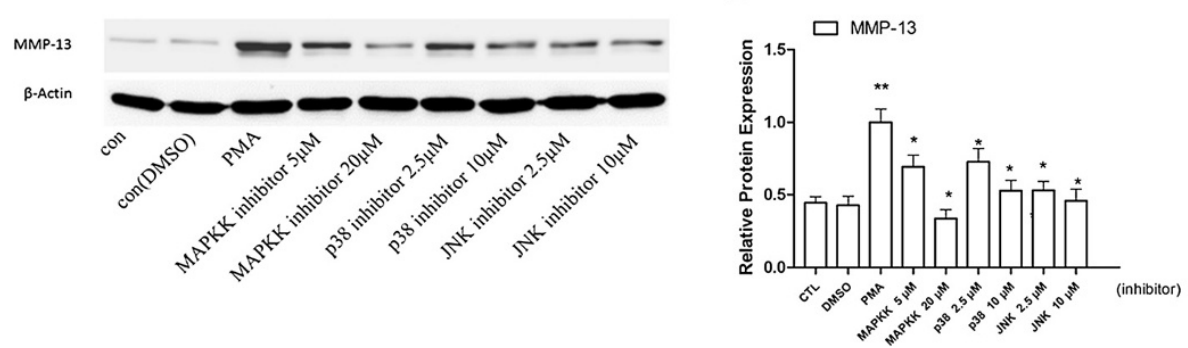

Figure 4 PMA induced EMMPRIN, MMP-9 and MMP13 expression by macrophages is blocked by a specific ERK, p38 and/or JNK inhibitors. A-B. The addition of a specific ERK inhibitor (PD98059, 5 and $20 \mu \mathrm{M}$ ), p38 inhibitor (SB203580, 2.5 and $10 \mu \mathrm{M}$ ) completely blocks PMA-induced MMP-9 protein expression and activation, and p38-specific inhibitor showed weaker function. ERK1/2 and p38-specific inhibitor inhibitor significantly decreased EMMPRIN expression, whereas JNK specific inhibitor showed no inhibitory effect. C-D. ERK1/2, p38 and JNK-specific inhibitor at high dose showed remarkable inhibitory effect on MMP-13 expression. ${ }^{*} P<0.05$ vs PMA group, $\# P>0.05$ vs PMA group.

determine whether AMPK inactivation promotes MMP9, MMP-13 and EMMPRIN expression. As shown in Figure 5A-C, inhibition AMPK by compound C (AMPK inhibitor) dramatically suppressed MMP-9, MMP-13 and EMMPRIN expression, indicating that AMPK chronic activation are important for PMA induced MMP-9, MMP-13 and EMMPRIN expression. Thus, inhibiting the activation of AMPK by curcumin (Figure 2C) may also contribute to attenuated MMP-9, MMP-13 and EMMPRIN expression. In addition, compound $C$ also reduced the phosphorylation of p38, JNK, and ERK in PMA induced THP-1 cells (Figure 4D-E), suggesting that the AMPK inhibitor diminished the activation of $\mathrm{p} 38$, JNK, and ERK pathways. Taken together, we concluded that curcumin significantly inhibited phosphorylation AMPK through MAPK pathways in dose-dependent manner, which led to down-regulated EMMPRIN and MMP-9 expression in PMA-induced THP-1 cells.

\section{Discussion}

In this study, our data support a novel effect of curcumin on the expression level of EMMPRIN, MMP-9 and MMP-13, suggesting that curcumin could be a potential therapeutic agent for ameliorating the development of atherosclerosis plaque. We found that curcumin inhibits EMMPRIN MMP-9 and MMP-13, expression via PKC and AMPK-dependent pathway in PMA induced THP-1 cells. Elevated expression and activity of MMP-13, MMP-9 and EMMPRIN are correlated with advanced atherosclerotic lesions followed by plaque rupture and myocardial infarction $[8,19,33,34]$, which can be inhibited by curcumin.

To elucidate the molecular mechanisms underlying anti-atherolsclerosis activity of curcumin in PMA induced THP-1 cells, we first measured the protein level of phosphorylated AMPK $\alpha$ in THP-1 differentiated macrophage. AMPK, the master regulator of energy metabolism, emerges as a kinase that controls glycogen utilization, lipid metabolism, fatty acid uptake and oxidation, and protein synthesis [35,36]. AMPK is also necessary for the invasive ability, the MMP-9 activity of THP-1 cells $[37,38]$, and PMA induced THP-1 cell adhesion to endothelial cells [39]. PMA has been shown to induce the activation of AMPK $\alpha$, and the inactivation of AMPK $\alpha$ resulted in down-regulation of MMP-9, MMP13 and EMMPRIN. As reported previously, Curcumin was shown to inhibit the activation of AMPK $\alpha$, although other research demonstrated different result $[40,41]$. The discrepancy may be due to different cell type and/or different inducing condition. However, no study has determined the role of curcumin in the long term activation of AMPK $\alpha$. In our study, we found that AMPK is activated during $48 \mathrm{~h}$ PMA induced cell differentiation, and curcumin suppresses the chronic activation of AMPKa in a dose-dependent manner. Consistent with our data, the activation of AMPKs has been reported to induce cell differentiation, including bone marrow-derived cells differentiation into endothelial cells [42] and osteoblastic 

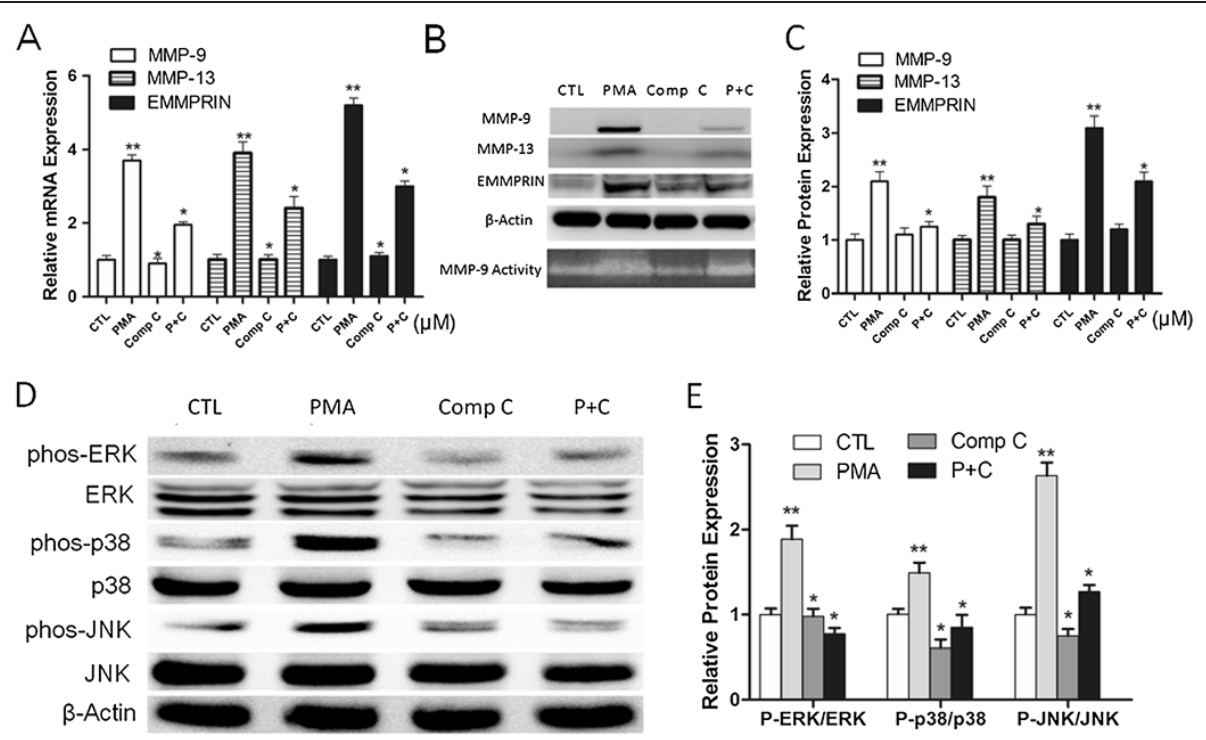

Figure 5 AMPK inhibitor mediated EMMPRIN, MMP-9 and MMP13 expression inhibition depends on the activation of MAPK pathway. A-C. Compound C, AMPK inhibitor, significantly inhibits EMMPRIN, MMP-9 and MMP13 expression in PMA induced THP-1 cells. Cells were pretreated with vehicle or Compound C (10 $\mu \mathrm{M})$ for $1 \mathrm{~h}$, followed by PMA for $48 \mathrm{~h}$. The mRNA level of EMMPRIN, MMP-9 and MMP13 was determined by qPCR (A), and protein level was determined by Western blot (B) and quantified by densitometric analysis (C). Comp C indicates group treated with compound $C ; P+C$ indicates group treated with both PMA and compound C. D-E. Compound C inhibited the activation of MAPK pathway. Differentiated THP-1 cells were treated with indicated agents, and assayed by Western blot using indicated antibodies (D) and quantified by densitometric analysis (E). ${ }^{*} P<0.05$ vs PMA group, ${ }^{* *} P<0.05$ vs $C T L$ group.

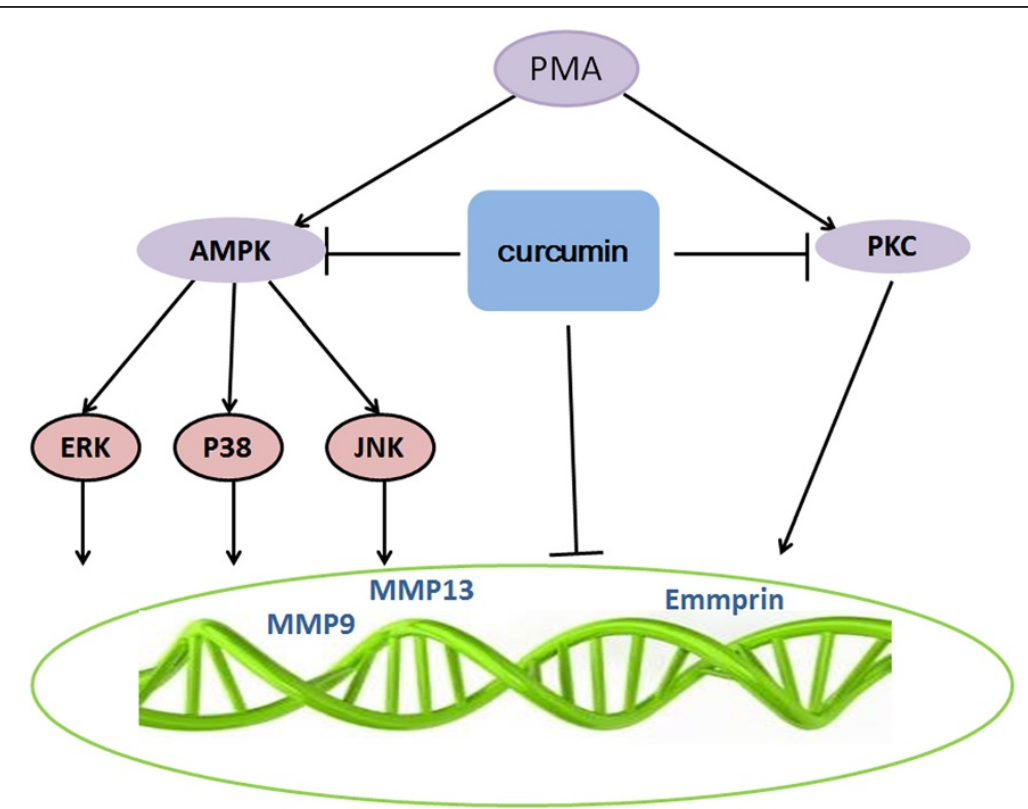

Figure 6 Model for regulation of MMP-9, MMP-13 and EMMPRIN by curcumin. PMA induced EMMPRIN, MMP-9 and MMP13 expression by macrophages through AMPK and PKC pathway.Curcumin attenuates expression of MMP-9, MMP-13 and EMMPRIN by inhibiting the activation of AMPK and PKC pathway. 
differentiation [43]. In addition, we observed that compound C (AMPK inhibitor) inhibits MMP-9, MMP-13 and EMMPRIN expression level in PMA induced THP-1 cell differentiation. PKC signal were actived during PMA induced cell differentiation and adhesion [31,32]. Our found showed PKC was actived by in PMA induced THP1 cells, curcumin can inhibit the activation of $\mathrm{PKC} \alpha$ and PKC $\beta 1$. Therefore, through inactivating AMPKs and PKC, curcumin decreases the MMP-9, MMP-13 and EMMPRIN level which results in inhibiting monocyte/macrophage differentiation.

In addition, compound $C$ also suppress the phosphorylation of three major classes of MAP kinase signaling (ERK, JNK, and p38), suggesting that curcumin may suppress MMP-9, MMP-13 and EMMPRIN level by inactivation of MAPK pathways. Previous data indicate that EMMPRIN and MMPs can be regulated by different factors, especially in MAPK pathways. For example, Lee et al. reported that MMP-9 production was enhanced in murine macrophages via activation of ERK and p38 MAPK [44,45]. Moreover, MMP-9, MMP13 and EMMPRIN level can be suppressed by ERK inhibitors or JNK siRNA [3,46,47]. Consistent with our previous studies $[28,29]$, MAPK cascades (p38, ERK1/2, and JNK) are activated to induce the expression of MMP-9 [48-50], MMP13 [51,52] and EMPRIN [53,54].

As shown in this study, PMA induced the phosphorylation of ERK1/2, p38 and JNK. Curcumin inhibits MAPKs phosphorylation, which contributes to the down-regulation of MMP-9, MMP-13 and EMMPRIN expression. This was further supported by the finding that the specific inhibitor of ERK1/2, p38 and JNK showed different extent in PMA induced protein expression. Similarly, we found that compound $C$ suppresses the phosphorylation of ERK1/2, p38 and JNK, and the expression of MMP-9 and EMMPRIN. All these results suggest that curcumin suppresses the activation of ERK1/2, p38 and JNK by inhibiting p-AMPK and PKC.

\section{Conclusion}

In summary, we showed that curcumin attenuates MMP-9, MMP-13 and EMMPRIN expression through the down-regulation of the AMPK and PKC pathway. (Figure 6). Moreover, we identified AMPK as a novel negative regulator of MMP-9 and EMMPRIN expression in THP-1 cell during differentiation. We also indicate that AMPK - MAPK and PKC pathways are involved in inhibiting MMP-9, MMP-13 and EMMPRIN expression. Because MMP-9 and MMP-13 plays an important role in the rupture of atheromatous plaques, our findings shed novel insight into the regulatory mechanism of MMP-9 and MMP-13 expression, the function of AMPK, and a potential treatment of atherosclerosis by curcumin.

\section{Competing interests}

The authors declare that they no competing interests.

\section{Authors' contributions}

$\mathrm{ZH}, \mathrm{CW}$ and JC design study, JC, ZH, LT, BY and ZH carried out the molecular studies, cell culture and drafted the manuscript. YF and KC perform the statistical analysis. WH design study and revised the manuscript. All authors read and approved the final manuscript.

\section{Funding}

This work was supported by the Chinese National Natural Science Foundation Grants (Grant No. 81270376, No. 81102837 and 81370224), project supported by the Natural Science Foundation of Zhejiang Province (Grant No. LY13H280004), the Shanghai Committee of Science and Technology of China (Grant No. 12ZR1419500), and Doctoral students innovation fund of Shanghai Jiaotong University School of Medicine (BXJ201229, BXJ201228). Research Fund for the Doctoral Program of Higher Education of China (20130072110016)

The funders had no role in study design, data collection and analysis, decision to publish, or preparation of the manuscript.

Received: 24 May 2014 Accepted: 16 September 2014

Published online: 21 September 2014

\section{References}

1. Joghetaei N, Stein A, Byrne RA, Schulz C, King L, May AE, Schmidt R: The Extracellular Matrix Metalloproteinase Inducer (EMMPRIN, CD147) - a potential novel target in atherothrombosis prevention? Thromb Res 2013, 131:474-480.

2. Zhu X, Song Z, Zhang S, Nanda A, Li G: CD147: a novel modulator of inflammatory and immune disorders. Curr Med Chem 2014, 21:2138-2145.

3. Kim JY, Kim WJ, Kim H, Suk K, Lee WH: The Stimulation of CD147 Induces MMP-9 Expression through ERK and NF-kappaB in Macrophages: Implication for Atherosclerosis. IImmune Netw 2009, 9:90-97.

4. Yang D, Wang J, Ni J, Shang S, Liu L, Xiang J, Li C: Temporal expression of metalloproteinase- 8 and -13 and their relationships with extracellular matrix metalloproteinase inducer in the development of ligatureinduced periodontitis in rats. J Periodontal Res 2013, 48:411-419.

5. Lizarbe TR, Tarin C, Gomez M, Lavin B, Aracil E, Orte LM, Zaragoza C: Nitric oxide induces the progression of abdominal aortic aneurysms through the matrix metalloproteinase inducer EMMPRIN. Am J Pathol 2009, 175:1421-1430

6. Schmidt R, Bultmann A, Ungerer M, Joghetaei N, Bulbul O, Thieme S, Chavakis T, Toole BP, Gawaz M, Schomig A, May AE: Extracellular matrix metalloproteinase inducer regulates matrix metalloproteinase activity in cardiovascular cells: implications in acute myocardial infarction. Circulation 2006, 113:834-841

7. Tang Y, Nakada MT, Kesavan P, McCabe F, Millar H, Rafferty P, Bugelski P, Yan L: Extracellular matrix metalloproteinase inducer stimulates tumor angiogenesis by elevating vascular endothelial cell growth factor and matrix metalloproteinases. Cancer Res 2005, 65:3193-3199.

8. Yoon YW, Kwon HM, Hwang KC, Choi EY, Hong BK, Kim D, Kim HS, Cho SH, Song KS, Sangiorgi G: Upstream regulation of matrix metalloproteinase by EMMPRIN; extracellular matrix metalloproteinase inducer in advanced atherosclerotic plaque. Atherosclerosis 2005, 180:37-44.

9. Vandooren J, Van den Steen PE, Opdenakker G: Biochemistry and molecular biology of gelatinase B or matrix metalloproteinase-9 (MMP-9): the next decade. Crit Rev Biochem Mol Biol 2013, 48:222-272.

10. Major TC, Liang L, Lu X, Rosebury W, Bocan TM: Extracellular matrix metalloproteinase inducer (EMMPRIN) is induced upon monocyte differentiation and is expressed in human atheroma. Arterioscler Thromb Vasc Biol 2002, 22:1200-1207.

11. Haug C, Lenz C, Diaz F, Bachem MG: Oxidized low-density lipoproteins stimulate extracellular matrix metalloproteinase Inducer (EMMPRIN) release by coronary smooth muscle cells. Arterioscler Thromb Vasc Biol 2004, 24:1823-1829.

12. Watanabe $\mathrm{N}$, Ikeda U: Matrix metalloproteinases and atherosclerosis. Curr Atheroscler Rep 2004, 6:112-120.

13. Shah PK, Falk E, Badimon JJ, Fernandez-Ortiz A, Mailhac A, Villareal-Levy G, Fallon JT, Regnstrom J, Fuster V: Human monocyte-derived macrophages induce collagen breakdown in fibrous caps of atherosclerotic plaques. 
Potential role of matrix-degrading metalloproteinases and implications for plaque rupture. Circulation 1995, 92:1565-1569.

14. Inokubo $Y$, Hanada $H$, Ishizaka $H$, Fukushi T, Kamada T, Okumura K: Plasma levels of matrix metalloproteinase-9 and tissue inhibitor of metalloproteinase- 1 are increased in the coronary circulation in patients with acute coronary syndrome. Am Heart J 2001, 141:211-217.

15. Gough PJ, Gomez IG, Wille PT, Raines EW: Macrophage expression of active MMP-9 induces acute plaque disruption in apoE-deficient mice. J Clin Invest 2006, 116:59-69.

16. Luttun A, Lutgens E, Manderveld A, Maris K, Collen D, Carmeliet P, Moons L: Loss of matrix metalloproteinase- 9 or matrix metalloproteinase- 12 protects apolipoprotein E-deficient mice against atherosclerotic media destruction but differentially affects plaque growth. Circulation 2004, 109:1408-1414.

17. Lee WH, Kim SH, Lee Y, Lee BB, Kwon B, Song H, Kwon BS, Park JE: Tumor necrosis factor receptor superfamily 14 is involved in atherogenesis by inducing proinflammatory cytokines and matrix metalloproteinases. Arterioscler Thromb Vasc Biol 2001, 21:2004-2010.

18. Tarin C, Gomez M, Calvo E, Lopez JA, Zaragoza C: Endothelial nitric oxide deficiency reduces MMP-13-mediated cleavage of ICAM-1 in vascular endothelium: a role in atherosclerosis. Arterioscler Thromb Vasc Biol 2009, 29:27-32.

19. Prescott MF, Sawyer WK, Von Linden-Reed J, Jeune M, Chou M, Caplan SL, Jeng AY: Effect of matrix metalloproteinase inhibition on progression of atherosclerosis and aneurysm in LDL receptor-deficient mice overexpressing MMP-3, MMP-12, and MMP-13 and on restenosis in rats after balloon injury. Ann N Y Acad Sci 1999, 878:179-190.

20. Kumar D, Kumar M, Saravanan C, Singh SK: Curcumin: a potential candidate for matrix metalloproteinase inhibitors. Expert Opin Ther Targets 2012, 16:959-972.

21. Min KJ, Um HJ, Cho KH, Kwon TK: Curcumin inhibits oxLDL-induced CD36 expression and foam cell formation through the inhibition of p38 MAPK phosphorylation. Food Chem Toxicol 2013, 58:77-85.

22. Coban D, Milenkovic D, Chanet A, Khallou-Laschet J, Sabbe L, Palagani A Vanden Berghe W, Mazur A, Morand C: Dietary curcumin inhibits atherosclerosis by affecting the expression of genes involved in leukocyte adhesion and transendothelial migration. Mol Nutr Food Res 2012, 56:1270-1281.

23. Yu YM, Lin HC: Curcumin prevents human aortic smooth muscle cells migration by inhibiting of MMP-9 expression. Nutr Metab Cardiovasc Dis 2010, 20:125-132.

24. Olszanecki R, Jawien J, Gajda M, Mateuszuk L, Gebska A, Korabiowska M, Chlopicki S, Korbut R: Effect of curcumin on atherosclerosis in apoE/LDLRdouble knockout mice. J Physiol Pharmacol 2005, 56:627-635.

25. Tsuchiya S, Kobayashi Y, Goto Y, Okumura H, Nakae S, Konno T, Tada K Induction of maturation in cultured human monocytic leukemia cells by a phorbol diester. Cancer Res 1982, 42:1530-1536.

26. Meng S, Cao JT, Zhang B, Zhou Q, Shen CX, Wang CQ: Downregulation of microRNA-126 in endothelial progenitor cells from diabetes patients, impairs their functional properties, via target gene Spred-1. J Mo/ Cell Cardiol 2012, 53:64-72.

27. Galis ZS, Sukhova GK, Lark MW, Libby P: Increased expression of matrix metalloproteinases and matrix degrading activity in vulnerable regions of human atherosclerotic plaques. J Clin Invest 1994, 94:2493-2503.

28. Huang Z, Wang L, Meng S, Wang Y, Chen T, Wang C: Berberine reduces both MMP-9 and EMMPRIN expression through prevention of $\mathrm{p} 38$ pathway activation in PMA-induced macrophages. Int $J$ Cardiol 2011, 146:153-158.

29. Huang Z, Wang C, Wei L, Wang J, Fan Y, Wang L, Wang Y, Chen T: Resveratrol inhibits EMMPRIN expression via P38 and ERK1/2 pathways in PMA-induced THP-1 cells. Biochem Biophys Res Commun 2008, 374:517-521.

30. Zhang J, Ge H, Wang C, Guo TB, He Q, Shao Q, Fan Y: Inhibitory effect of PPAR on the expression of EMMPRIN in macrophages and foam cells. Int J Cardiol 2007, 117:373-380.

31. Besson A, Davy A, Robbins SM, Yong WW: Differential activation of ERKs to focal adhesions by PKC epsilon is required for PMA-induced adhesion and migration of human glioma cells. Oncogene 2001, 20:7398-7407.

32. Kim MS, Lim WK, Cha JG, An NH, Yoo SJ, Park JH, Kim HM, Lee YM: The activation of PI 3-K and PKC zeta in PMA-induced differentiation of HL-60 cells. Cancer Lett 2001, 171:79-85.
33. Newby AC: Metalloproteinase expression in monocytes and macrophages and its relationship to atherosclerotic plaque instability. Arterioscler Thromb Vasc Biol 2008, 28:2108-2114.

34. Cimmino G, Ragni M, Cirillo P, Petrillo G, Loffredo F, Chiariello M, Gresele P, Falcinelli $E$, Golino P: C-reactive protein induces expression of matrix metalloproteinase-9: a possible link between inflammation and plaque rupture. Int J Cardiol 2013, 168:981-986.

35. Blagih J, Krawczyk CM, Jones RG: LKB1 and AMPK: central regulators of lymphocyte metabolism and function. Immunol Rev 2012, 249:59-71.

36. Fullerton MD, Steinberg GR: SIRT1 takes a backseat to AMPK in the regulation of insulin sensitivity by resveratrol. Diabetes 2010, 59:551-553.

37. Chen H, Liu X, Chen H, Cao J, Zhang L, Hu X, Wang J: Role of SIRT1 and AMPK in mesenchymal stem cells differentiation. Ageing Res Rev 2014, 13:55-64.

38. Chuang SY, Yang SH, Chen TY, Pang JH: Cilostazol inhibits matrix invasion and modulates the gene expressions of MMP-9 and TIMP-1 in PMA-differentiated THP-1 cells. Eur J Pharmacol 2011, 670:419-426.

39. Chang MY, Huang DY, Ho FM, Huang KC, Lin WW: PKC-dependent human monocyte adhesion requires AMPK and Syk activation. PLOS One 2012, 7:e40999.

40. Pu Y, Zhang H, Wang P, Zhao Y, Li Q, Wei X, Cui Y, Sun J, Shang Q, Liu D, Zhu Z: Dietary curcumin ameliorates aging-related cerebrovascular dysfunction through the AMPK/uncoupling protein 2 pathway. Cell Physiol Biochem 2013, 32:1167-1177.

41. Xiao K, Jiang J, Guan C, Dong C, Wang G, Bai L, Sun J, Hu C, Bai C: Curcumin induces autophagy via activating the AMPK signaling pathway in lung adenocarcinoma cells. J Pharmacol Sci 2013, 123:102-109.

42. Zhu Z, Fu C, Li X, Song Y, Li C, Zou M, Guan Y, Zhu Y: Prostaglandin E2 promotes endothelial differentiation from bone marrow-derived cells through AMPK activation. PLoS One 2011, 6:e23554.

43. Kimdo Y, Park KH, Jung MS, Huang B, Yuan HD, Quan HY, Chung SH: Ginsenoside Rh2(S) induces differentiation and mineralization of MC3T3-E1 cells through activation of the PKD/AMPK signaling pathways. Int J Mol Med 2011, 28:753-759.

44. Lee SJ, Kim CE, Yun MR, Seo KW, Park HM, Yun JW, Shin HK, Bae SS, Kim CD: 4-Hydroxynonenal enhances MMP-9 production in murine macrophages via 5-lipoxygenase-mediated activation of ERK and p38 MAPK. Toxicol Appl Pharmacol 2010, 242:191-198.

45. Park D, Shim E, Kim Y, Kim YM, Lee H, Choe J, Kang D, Lee YS, Jeoung D: C-FLIP promotes the motility of cancer cells by activating FAK and ERK, and increasing MMP-9 expression. Mol Cells 2008, 25:184-195.

46. Byun HJ, Hong IK, Kim E, Jin YJ, Jeoung DI, Hahn JH, Kim YM, Park SH, Lee H: A splice variant of CD99 increases motility and MMP-9 expression of human breast cancer cells through the AKT-, ERK-, and JNK-dependent AP-1 activation signaling pathways. J Biol Chem 2006, 281:34833-34847

47. Yu T, Wu Y, Helman Jl, Wen Y, Wang C, Li L: CXCR4 promotes oral squamous cell carcinoma migration and invasion through inducing expression of MMP-9 and MMP-13 via the ERK signaling pathway. Mol Cancer Res 2011, 9:161-172.

48. Simon C, Goepfert H, Boyd D: Inhibition of the p38 mitogen-activated protein kinase by SB 203580 blocks PMA-induced Mr 92,000 type IV collagenase secretion and in vitro invasion. Cancer Res 1998, 58:1135-1139.

49. Park MJ, Park IC, Hur JH, Kim MS, Lee HC, Woo SH, Lee KH, Rhee $\mathrm{CH}$, Hong $\mathrm{SI}$, Lee SH: Modulation of phorbol ester-induced regulation of matrix metalloproteinases and tissue inhibitors of metalloproteinases by SB203580, a specific inhibitor of p38 mitogen-activated protein kinase. J Neurosurg 2002, 97:112-118.

50. Weng CJ, Chau CF, Hsieh YS, Yang SF, Yen GC: Lucidenic acid inhibits PMA-induced invasion of human hepatoma cells through inactivating MAPK/ERK signal transduction pathway and reducing binding activities of NF-kappaB and AP-1. Carcinogenesis 2008, 29:147-156.

51. Tan TW, Yang WH, Lin YT, Hsu SF, Li TM, Kao ST, Chen WC, Fong YC, Tang CH: Cyr61 increases migration and MMP-13 expression via alphavbeta3 integrin, FAK, ERK and AP-1-dependent pathway in human chondrosarcoma cells. Carcinogenesis 2009, 30:258-268.

52. Mengshol JA, Vincenti MP, Brinckerhoff CE: IL-1 induces collagenase-3 (MMP-13) promoter activity in stably transfected chondrocytic cells: requirement for Runx-2 and activation by p38 MAPK and JNK pathways. Nucleic Acids Res 2001, 29:4361-4372. 
53. Lim M, Martinez T, Jablons D, Cameron R, Guo H, Toole B, Li JD, Basbaum C: Tumor-derived EMMPRIN (extracellular matrix metalloproteinase inducer) stimulates collagenase transcription through MAPK p38. FEBS Lett 1998, 441:88-92.

54. Zhou J, Zhu P, Jiang JL, Zhang Q, Wu ZB, Yao XY, Tang H, Lu N, Yang Y, Chen ZN: Involvement of CD147 in overexpression of MMP-2 and MMP-9 and enhancement of invasive potential of PMA-differentiated THP-1. BMC Cell Biol 2005, 6:25.

doi:10.1186/s12967-014-0266-2

Cite this article as: Cao et al.: Curcumin inhibits EMMPRIN and MMP-9 expression through AMPK-MAPK and PKC signaling in PMA induced macrophages. Journal of Translational Medicine 2014 12:266.

\section{Submit your next manuscript to BioMed Central and take full advantage of:}

- Convenient online submission

- Thorough peer review

- No space constraints or color figure charges

- Immediate publication on acceptance

- Inclusion in PubMed, CAS, Scopus and Google Scholar

- Research which is freely available for redistribution 